\title{
Entwicklung und Einsatz von AR-Anwendungen in der Hochschule am Beispiel des Projekts „Augmented Learning“ an der Universität Duisburg-Essen
}

\author{
Mirco Zick (ID - Marianne Wefelnberg
}

Eingegangen: 18. Juli 2021 / Angenommen: 19. November 2021 / Online publiziert: 13. Dezember 2021

(C) Der/die Autor(en) 2021

Zusammenfassung Die Einsatzmöglichkeiten von Augmented Reality (AR) im Bildungsbereich werden bereits seit längerer Zeit diskutiert. Betrachten wir den konkreten Einsatz entsprechender Anwendungen in der Praxis, kann zumindest für den Hochschulbereich festgestellt werden, dass sich dort AR noch nicht in der Breite durchgesetzt hat. Im vorliegenden Artikel werden mögliche Gründe aufgezeigt, warum es aktuell noch wenige Praxisbeispiele in der Hochschule gibt und welche pädagogischen Konzepte sich zur näheren Untersuchung von AR und ihrer Potenziale für die Bildung heranziehen lassen. Im Anschluss wird ein Projekt an der Universität Duisburg-Essen (UDE) vorgestellt, in dem eine Augmented-LearningApp entwickelt wurde. Mithilfe der App können Lehrende zukünftig 3-D-Modelle konzipieren und in ihrer Veranstaltung einsetzen. Der Artikel zeigt zudem die Entscheidungskriterien auf, die zu einer internen Entwicklung der AR-App führten.

Schlüsselwörter Augmented Reality · Lehr-/Lerninnovationen ·

Technologieentwicklung $\cdot$ AR-App $\cdot$ Hochschule

Mirco Zick $(\varangle) \cdot$ Marianne Wefelnberg

Geschäftsbereich Lerntechnologien, Zentrum für Informations- und Mediendienste, Essen,

Deutschland

E-Mail: mirco.zick@uni-due.de

Universität Duisburg-Essen, Essen, Deutschland 


\title{
Developing Augmented Reality Applications for Universities Exemplified by the Project "Augmented Learning" at the University of Duisburg-Essen
}

\begin{abstract}
The discussion about Augmented Reality (AR) in education is not new. Nonetheless, using AR in education is not very common, particularly not at German universities. The following article addresses the reasons, why there are only a few practical approaches using AR in education. After giving an overview of the difficulties to introduce digital tools into the university, the potentials and sticking points of AR are considered. Subsequently a short assessment of the pedagogical concepts which may be useful to examine the additional value of AR in education is given. In the second part a AR project at the University of Duisburg-Essen (UDE) called "Augmented Learning App" is described. Via the AR-App lecturers are able to build and present 3-D-Models and use them in their lessons. Within this article the process of establishing and implementing the AR-App at the UDE is given and how common obstacles were handled.
\end{abstract}

Keywords Augmented Reality · Innovation in education · Technical development · AR-App · University

\section{Einleitung}

Die Digitalisierung in Bildungsinstitutionen hat mittlerweile einen festen Platz als eigenständiges Thema im Bereich Lehren und Lernen. Laut dem Gutachten der Expertenkommission Forschung und Innovation (EFI) aus dem Jahr 2019 steigt der Einsatz digitaler Lehr- und Lernformate an deutschen Hochschulen jedoch eher langsam an. Die nur schleppende Umsetzung digitaler Lehr- und Lernformate hat mehrere Gründe. Zum einen werden Innovationen überwiegend mittels externer Projektförderungen umgesetzt. Dadurch fehlen dauerhafte Strukturen sowie die personellen und infrastrukturellen Ressourcen, um eigene Systeme zu entwickeln oder zu administrieren (Hochschulforum Digitalisierung 2016). Im Resultat gehen das Knowhow der Projektmitarbeitenden und damit u. U. auch die Produkte nach Abschluss eines Projekts verloren und werden nicht nachhaltig in der Hochschule verankert (Seufert und Euler 2004). Zum anderen bedeutet die Nutzung digitaler Medien in der Lehre auch einen Kulturwandel für die Hochschulen und ihre Akteure.

Ein wichtiger Motor für diesen Kulturwandel sind die Lehrenden, die die neuen Technologien einsetzen. Schon 2005 wies Euler darauf hin, dass Lehrende nicht dazu verpflichtet seien, digitale Medien in ihre Lehre zu integrieren und sie den Einsatz daher häufig eher vermieden. Die Nutzung neuer Technologien löse bei ihnen Unsicherheit aus und erfordere die Änderung von Lehrgewohnheiten. Der Unsicherheit begegneten viele Hochschulen mit der Gründung von Servicezentren. So gaben $90 \%$ der von der EFI (2019) befragten Hochschulen an, „E-LearningZentren zur Unterstützung der Lehrenden und zur Erarbeitung digitaler Lerninhalte zu betreiben“. Die Lehrenden empfinden die dort aufgesetzten Angebote laut der Befragung jedoch häufig als nicht ausreichend. 
Die beiden Punkte der Projekthaftigkeit von Innovationen und des Kulturwandels verdeutlichen zugleich das Spannungsverhältnis zwischen der Förderung innovativer Forschungs- und Entwicklungsaufgaben zur Digitalisierung an Hochschulen und der Bereitstellung möglichst niedrigschwelliger Angebote für einen breitenwirksamen Einsatz durch mit digitaler Lehre bisher wenig vertraute Lehrende (Hochschulforum Digitalisierung 2016; Kleimann und Wannemacher 2004). Aus diesem Grund ist es für die erfolgreiche und breit angelegte Implementierung (neuer) digitaler Medien in der Lehre essentiell, Lehrende an den Umgang mit digitalen Technologien heranzuführen, einen didaktisch sinnvollen Einsatz aufzuzeigen und sie beratend zu begleiten. Dies bedeutet wiederum die Notwendigkeit, dauerhaft und in ausreichendem Umfang qualifiziertes Personal für den technischen und didaktischen Support einzustellen.

Ein weiterer Aspekt für den zögerlichen Einsatz von digitalen Medien in der Lehre liegt laut Reinmann (2005) in der mangelnden Anerkennung innovativer Lehre. Es sei damit nicht per se ein Reputationsgewinn verbunden. Die EFI kommt in ihrem Gutachten noch 2019 zu dem Schluss, dass die Lehre kein primäres Qualitätsmerkmal zur Bewertung des wissenschaftlichen Personals und der Hochschule darstelle und weiterhin passende Anreize fehlen, die insbesondere auch deputatsrechtliche Unsicherheiten minimieren (EFI 2019).

Im folgenden Artikel wird der Einsatz von AR-Anwendungen in der Hochschule betrachtet, und die Potenziale von AR sowie die Herausforderungen bei der Implementierung anhand von praktischen Erfahrungen und Marktbeobachtungen werden vorgestellt. Bereits im NMC Horizon Report 2016 (Johnson et al. 2016) wird AR als Trend aufgeführt, der zu dieser Zeit so eingeschätzt wurde, dass er mittelfristig in einem Zeitraum von zwei bis drei Jahren für Universitäten an Bedeutung gewinnt.

Diese Prognose ist bisher nicht eingetreten. Virtual und Augmented Reality und ihre Potenziale für die Bildung werden zwar seit Jahren diskutiert, haben sich aber bis heute nicht flächendeckend durchgesetzt. Trotzdem sind Überlegungen zu AR als mehrwertschaffende Technologie im Bildungsbereich nicht verschwunden. Im Gegenteil: Zunehmend werden (Forschungs-)Projekte mit diesem Schwerpunkt ausgeschrieben und gefördert. ${ }^{1}$ Die weiter oben beschriebenen Hürden bei der Implementierung einer innovativen Technologie kommen bei AR u. a. aufgrund der aufwendigen Erstellung der 3-D-Modelle besonders zum Tragen. Daher wird der Fokus im Folgenden auf diesen Hürden und etwaigen Problemlösungsansätzen liegen und anhand eines Projekts der Universität Duisburg-Essen verdeutlicht.

\footnotetext{
1 Zu nennen sind hier beispielsweise Projekte wie AR/VR.nrw (https://vrar.uni-wuppertal.de/de/forschunglehre/projektvorhaben.html, abgerufen am 18. Juli 2021), welches auf Landesebene in der Förderlinie Digitale Werkzeuge für die Hochschullehre im Rahmen der Digitalen Hochschule NRW (DH NRW) durchgeführt wird oder Förderprogramme des Bundesministeriums für Bildung und Forschung (BMBF) im Aus- und Weiterbildungsbereich, z. B. HandLeVR (https://www.uni-due.de/e-learning/ag-xr-learning. php\#handlevr, abgerufen am 18. Juli 2021).
} 


\section{Potenziale und Herausforderungen}

AR wird definiert als ,,an emerging form of experience in which the real world (RW) is enhanced by computer-generated content which is tied to specific locations and/or activities." (Yuen et al. 2011). Wu et al. (2013) heben drei Kategorien bezüglich des Designs einer AR-Anwendung im Bildungskontext hervor, die auch Auswirkungen auf die Prozesse der Erstellung einer AR-Anwendung haben: (1) die Einnahme einer Rolle durch den Lernenden, (2) die Verankerung mit dem Ort und (3) die Bearbeitung einer Aufgabe. Die Kategorien werden dabei nicht immer strikt voneinander getrennt angewendet und es gibt Überschneidungen hinsichtlich der Präsentationsformate oder des didaktischen Settings.

Durch die mobile Nutzung von AR-Anwendungen besteht die Möglichkeit, dass Lernende eine bestimmte Perspektive oder Rolle einnehmen und mit anderen Lernenden in Kontakt treten, z.B. indem sie Daten austauschen oder Informationen sammeln und analysieren (1). Informationen können dabei direkt vor Ort abgerufen werden (2). Diese Form wird beispielsweise für die Veranschaulichung an historisch bedeutsamen Orten eingesetzt. Mithilfe von AR-Modellen ist es möglich, Gebäude oder Landschaften zu zeigen, die nicht mehr existieren und darüber Authentizität in das Lernerlebnis einzubringen. Die Bearbeitung einer Aufgabe kann entweder in den Kategorien (1) und (2) integriert sein oder die AR-Anwendung selbst ist als Aufgabe gestaltet, z. B. indem Lernende mithilfe der Applikation eine physikalische Gesetzmäßigkeit erkunden sollen (3). Mit den Kategorien, wie sie Wu et al. (2013) anwenden, lassen sich entsprechende didaktische Settings verbinden, in denen ARAnwendungen einen Mehrwert bieten können. Nach Bower et al. (2014) werden folgende lernpsychologische Ansätze in Verbindung mit AR diskutiert: Konstruktivismus, Situatives Lernen, Game-based Learning und Forschungsbasiertes Lernen.

In diesem Kontext lohnt sich ein Blick auf pädagogische Ansätze des Instructional Designs, um Potenziale von AR-Anwendungen zu beleuchten. Bei der Cognitive Load Theory (Sweller 1994) werden drei Faktoren definiert, die die kognitive Verarbeitung neuer Informationen im Arbeitsgedächtnis beeinflussen: (1) Intrinsic Cognitive Load, (2) Extraneous Cognitive Load und (3) Germane Cognitive Load. Es wird davon ausgegangen, dass Lernende nur eine begrenzte Anzahl von neuen Informationen (zeitgleich) im Arbeitsgedächtnis verarbeiten können. Je nach Komplexität des Lerngegenstandes und Vorwissens des Lernenden wird das Arbeitsgedächtnis entsprechend belastet (Intrinsic Cognitive Load). Der Extraneous Cognitive Load meint die Ablenkung von lernrelevanten Inhalten durch überflüssige Informationen. Er bezieht sich z. B. auf die Art der Präsentation des Lerngegenstandes. Der Germane Cognitive Load bezieht sich schließlich auf kognitiven Verarbeitungsprozesse und darauf, wie Lerninhalte organisiert und in bestehendes Wissen integriert werden. In Zusammenhang mit den Erkenntnissen aus der Dual Coding Theory (Paivio 1986) und der Cognitive Theory of Multimedia Learning (Mayer 2001) verringert sich der Extraneous Cognitive Load, sobald Lernmaterialien sowohl den verbalen als auch den visuellen Verarbeitungskanal des Gehirns ansprechen. Mayer (2001) spricht dabei vom modality principle. Über eine AR-App wäre es entsprechend möglich, Inhalte sowohl visuell aufzubereiten, als auch verbal mithilfe eines Voiceovers zu erläutern. Laut Mayer wird der Extraneous Cognitive Load auch durch das 
so genannte temporal contiguity principle verringert, welches besagt, dass Wörter und Bilder dem Lernenden idealerweise gleichzeitig präsentiert werden sollten: „the learner is more likely to be able to build mental connections between verbal and visual representations. When corresponding portions of narration and animation are separated in time, the learner is less likely to be able to hold mental representations of both in working memory at the same time and thus less likely to be able to build mental connections between verbal and visual representations." (Mayer 2001) Auch hier bieten AR-Anwendungen neue Möglichkeiten zur Umsetzung des temporal contiguity principle. Untersuchungen zur Reduzierung des Extraneous Cognitive Load beim Einsatz von Augmented- und Virtual-Reality-Anwendungen in der Lehre kamen jedoch zu unterschiedlichen Ergebnissen (Thees et al. 2020; Radu 2014). Die Erkenntnisse sollten dabei allerdings differenziert nach Lerngegenstand, Form der AR-Anwendung, Vorwissen der Lernenden etc. betrachtet werden. Es lässt sich aber vermuten, dass der Einsatz von AR-Anwendungen - ähnlich wie bei anderen digitalen Lernangeboten - nicht per se einen Mehrwert darstellt (Thees et al. 2020).

Ein Vorteil von mobilen AR-Anwendungen besteht darin, dass sie durch den technologischen Fortschritt mobiler Endgeräte nun einer größeren Anzahl von Menschen zur Verfügung stehen und für das eigene Lernen eingesetzt werden können (Niedermeier und Müller-Kreiner 2019). Insbesondere AR bietet hier die Möglichkeit, Studierende aktiv in die Lehrveranstaltung einzubinden und situative Lernszenarien zu schaffen (Joan 2015; Bower et al. 2014).

Den Potenzialen beim Einsatz von AR-Anwendungen im Bildungsbereich steht die teils aufwändige Konzeption und der Produktionsaufwand von AR-Anwendungen gegenüber. Je umfangreicher die Funktionalitäten der AR-Anwendung sein sollen, desto mehr Kompetenzen sind für die Anwendungsentwicklung nötig. Damit AR-Anwendungen von vielen Lernenden genutzt werden können, muss die Entwicklung mit unterschiedlichen Betriebssystemen und Endgeräten kompatibel sein und ansprechende und fachlich korrekte 3-D-Modelle beinhalten. Dies setzt also eine Zusammenarbeit von Expert:innen unterschiedlicher Fachdisziplinen sowie der Informatik, Pädagogik und des Grafikdesign voraus (Zender et al. 2018).

Lehrende benötigen ferner eine konkrete Vorstellung davon, welchen Mehrwert sie durch die Nutzung von AR in ihrer Lehrveranstaltung sehen. Wie bereits in der Einleitung beschrieben ist für die Einführung von digitalen Technologien in die Lehre ein Kulturwandel seitens der Lehrenden erforderlich. Zur Heranführung von Lehrenden an neue Technologien gelten weiterhin Schulungen und Workshops als Mittel der Wahl. Sie haben i.d. R. zum Ziel, Lehrenden aufzuzeigen, wie sie Systeme oder digitale Tools in didaktisch sinnvoller Weise eigenständig in ihre Veranstaltung einbinden können. Bei AR ist die eigenständige Umsetzung durch Lehrende nur begrenzt möglich, da die Erstellung passender Modelle sehr komplex und der Aufwand entsprechend hoch sein kann. Vielen Lehrenden fehlen die entsprechenden Fähigkeiten oder zeitlichen Ressourcen zur Umsetzung von AR-Anwendungen. Sie sind daher auf Expert:innen aus der IT angewiesen, die sie bei der Realisierung der AR-Projekte unterstützen. Auch rechtliche Fragen z.B. in Bezug auf die Nutzung einer App können Gründe für eine eher zurückhaltende Position gegenüber AR sein. 
Im folgenden Kapitel sollen zunächst die technischen Anforderungen zur Realisierung einer AR-App betrachtet werden, bevor dann auf die konkrete Umsetzung an der UDE eingegangen wird.

\section{Implikationen zur technischen Realisierung}

Wie zuvor beschrieben sind die Faktoren mannigfaltig, welche den Kontext sowie die zugrundeliegende Intention des Einsatzes von AR-Technologie im Bildungsbereich definieren können. Aus den vielen möglichen Einsatzszenarien und den daraus abgeleiteten Anforderungen an eine AR-Anwendung resultiert der Bedarf an theoretisch unzähligen Produkten. Nur über die Bereitstellung individuell anpassbarer Anwendungen könnten eine entsprechende Anzahl von Lernangeboten erarbeitet und entsprechende Ressourcen bereitgestellt werden.

Wie bereits zu Beginn aufgezeigt wurde, können einzelne Bildungseinrichtungen einen solchen individuellen und produktorientierten Lösungsansatz u. a. aufgrund der Förderstrukturen kaum leisten. Ein nachhaltiger Betrieb über den Projektzeitraum hinaus wird dadurch erschwert. Auch Unternehmen, die AR-Anwendungen anbieten, können ein dauerhaft auf die Bedarfe von Hochschulen ausgerichtetes Produkt- und Serviceangebot häufig nicht liefern. So ist auf beiden Seiten die Wirtschaftlichkeit im jeweiligen Handeln kritisch durch eine Abwägung der Kosten durch Entwicklung und Support (Lee 2012) im Vergleich zum generierten Gewinn zu hinterfragen.

Um eine breite fachliche Anwendbarkeit von AR-Anwendungen in der Hochschullehre zu gewährleisten, ist die Wahl eines möglichst generisch einsatzfähigen AR-Systems ratsam. Die damit einhergehenden Aspekte der Nachhaltigkeit und Wirtschaftlichkeit können eine Entscheidungsgrundlage für Hochschulen bilden, das eigene Lehrportfolio mithilfe von AR-Technologie weiterzuentwickeln.

Je populärer AR-Technologien insgesamt werden, desto größere Dynamiken stellen sich auf dem Markt ein. Dies bedeutet für Nutzer:innen eine zunehmende Unsicherheit hinsichtlich der dauerhaften Verfügbarkeit von Produkten. Beispielhaft lässt sich dies an der Übernahme zahlreicher auf AR spezialisierter Startups aufzeigen, die von so genannten ,Techriesen“" aufgekauft wurden (z.B. Metaio von Apple in $2015^{2}$ ). In der Konsequenz stehen teils weit verbreitete AR-Lösungen nicht mehr zur Verfügung und bereits erstellte Lehr-Lerninhalte sind ad hoc nicht mehr zugänglich.

Auf der Ebene der Lehrenden liegt die Entscheidungsrelevanz hingegen in der Fähigkeit zur inhaltlichen Adaption und Integration in das eigene didaktische Setting. Viele am Markt befindliche Softwarelösungen sind primär auf die reine Umsetzung bildgestützter AR ausgelegt. D.h., dass zuvor deklarierte visuelle Marker (z. B. QRCodes oder Abbildungen) mit vornehmlich dreidimensionalen Objekten verknüpft werden. Die spätere Anwendung wird dann bei der Erkennung dieser Marker zur überlagerten Anzeige des 3-D-Objekts in die reale Welt getriggert. Die meisten Bestandssysteme auf dem Markt sind jedoch auf die reine Objekt- bzw. Produktvisualisierung ausgelegt. Im Kern ermöglicht dies sicherlich eine niedrigschwellige

\footnotetext{
2 Siehe z.B.: https://www.theverge.com/2015/5/28/8682505/apple-acquires-metaio-augmented-realitystartup/ (abgerufen am 30.10.2021).
} 
Realisierung einer AR-Anwendung, wird jedoch didaktischen Anforderungen nicht gerecht oder erschwert gar die Integration der Technologie. Um dies zu vermeiden, müssten ausgehend von einer initialen Repräsentation (z. B. 3-D-Struktur) vertiefende Informationen, abhängig von dem mobilen Endgerät, direkt an diese gekoppelt werden können. Dies ermöglicht es den Lernenden nicht nur, das Objekt visuell zu erkunden, sondern erleichtert ihnen die Transferleistung in die physische Welt. Eine Umsetzung mithilfe mehrerer Marker erscheint hierbei unübersichtlich und nicht zielführend. Alternativ könnten entsprechende Zusatzinformation auf andere Plattformen ausgelagert werden, was jedoch einen Medienbruch bedeuten würde. Integrierte Funktionen für die Bereitstellung von Assessments bzw. zur Überprüfung des Wissensstandes, sind in der Regel nicht vorgesehen.

Neben dieser eher funktionalen Perspektive, bestehen die Herausforderungen auch in der Erstellung von 3-D-Modellen, wie sie in Kap. 2 bereits erwähnt wurden. So müssen für (mobile) AR-Anwendungen die Inhalte erst noch produziert oder für das Endgerät angepasst werden. Primär bilden hier die im Fokus stehenden 3-D-Objekte eine Herausforderung für Lehrende, da es kaum adäquate Anlaufstellen zur Nutzung lizenzfreier 3-D-Modelle gibt. Derartige Modellierungsarbeiten erfordern eine hohe interdisziplinäre Expertise, die entweder aus persönlichem Interesse heraus aufgebaut oder durch entsprechende Fachkräfte aus Designabteilungen oder Agenturen abgedeckt werden muss.

Zuletzt sollte der Einsatz von AR-Lösungen im Bildungsbereich auch aus juristischer Perspektive kritisch reflektiert werden. Insbesondere die auf dem Markt ,frei nutzbaren“, also unentgeltlichen Produkte finanzieren sich, häufig mit der aus Nutzungsdaten generierten In-App-Werbung. Der vermehrte Einsatz von Cloud-Computing-Modellen ist hinsichtlich des Datenschutzes ebenso kritisch zu betrachten, tangiert aber auch Themen wie das Urheber- bzw. Verwertungsrecht. So ist der Großteil entsprechender AR-Angebote bzw. -Services auf meist nicht (mehr) nachvollziehbaren Server-Infrastrukturen ausgelagert.

\section{Entwicklung einer generischen AR-Anwendung am Beispiel des Projekts ,Augmented Learning““}

Im Sommer 2017 wurde im Rahmen einer Fachkonferenz ein Dozent der UDE auf die AR-Anwendung „Planktomania AR“3 französischer Kolleg:innen aufmerksam. Der Dozent sah in der AR-Anwendung Potenziale für seine eigene Lehre und wandte sich an die Mitarbeitenden des ZIM der UDE, um Möglichkeiten für ein vergleichbares Vorhaben zu sondieren. Aufgrund der bereits vorhandenen Governance-

\footnotetext{
3 Planktomania AR ist eine Augmented-Reality Anwendung für mobile Endgeräte. Sie wurde durch die meeresbiologische und meereskundliche Forschungs- und Lehreinrichtung Station biologique de Roscoff initiiert. Weitere Informationen zur Anwendung sind unter dem nachfolgenden Link zu finden: https:// planktomania.org (abgerufen am 15.07.2021).
} 
Strukturen, welche u.a. im Rahmen der Strategie zur Digitalisierung in Studium und Lehre $^{4}$ aufgebaut wurden, konnte auf das Vorhaben eingegangen werden.

Erfahrungsgemäß stellen (fachspezifische) Differenzierungen von Mikro- oder auch Nanostrukturen insbesondere Studienanfänger:innen vor eine große Herausforderung. Derartige Strukturen werden meist nur über zweidimensionale, statische Abbildungen präsentiert und sind damit zur selbstgesteuerten Exploration eines eigentlich dreidimensionalen Objekts nur eingeschränkt geeignet. Die notwendige Transferleistung der Studierenden ist entsprechend hoch und kann die extrinsische kognitive Belastung des Arbeitsgedächtnisses erhöhen (siehe Kap. 2 Extraneous Cognitive Load). Um den Facheinstieg zu erleichtern und gleichzeitig einen motivierenden Fokus auf die eigentlichen Fach- bzw. Lerninhalte zu legen, sollten die sonst schwer greifbaren Strukturen deshalb visuell und zugleich interaktiv in die reale Welt projiziert werden (Zick o.J.).

Damit die AR-Anwendung erfolgreich und über Fächergrenzen hinweg eingesetzt werden kann, wurde zunächst eine Anforderungserhebung durchgeführt. Damit wurde die Lauffähigkeit der künftigen AR-App auf den gängigen Betriebssystemen und ein generisch einsetzbares Funktionsspektrum innerhalb der App sichergestellt (Zick o.J.). In Ermangelung entsprechender Ausschreibungen wurde das Projekt Ende 2017 als internes (Kooperations-) Vorhaben gestartet und bislang ausschließlich über Haushaltsmittel bestritten. Durch die Realisierung des Projekts mithilfe von Bestandspersonal verbleibt die technische Expertise an der UDE und sichert eine hohe Flexibilität hinsichtlich der Inhalte und Funktionalitäten. Mit dem Verzicht auf die Verwendung externer Bestandsprodukte wurde darüber hinaus das bereits oben beschriebene Risiko vermieden, durch einen Aufkauf eines Anbieters nicht mehr auf bereits erstellte Lerninhalte sowie -infrastrukturen zugreifen zu können. Eine damalige Marktrecherche zeigte zudem, dass kein bestehendes Produkt die benötigten Anforderungen hätte erfüllen können. Für die technische Realisierung wurde die bereits in der Branche etablierte Unity3D-Engine gewählt, welche die Software auch auf den gängigen Betriebssystemen im mobilen Sektor ausliefern kann. Die AR-Implementierung wurde mittels der Software Vuforia umgesetzt. Beide Entwicklungskomponenten weisen für ein solches Vorhaben geeignete Lizenzmodelle auf.

Hinsichtlich folgender Eigenschaften und Funktionen wurden folgende Musthaves definiert: Die App soll auf Android und iOS lauffähig sein, da diese den überwiegenden Anteil der mobilen Endgeräte ausmachen. Beim Starten der App soll eine (optionale) „Einführung“ angeboten werden, welche (1) die Anwendung als Ganzes sowie (2) die jeweilige Lehrveranstaltung näher erläutert. Anschließend kann über die Kamera des Endgeräts ein beliebiger Marker (QR-Code, Abbildung o. ä.) erfasst werden, welcher das initiale AR-Objekt (i.d.R. 3-D-Modelle) in die reale Umgebung projiziert. Mit diesem kann dann über den Marker (Rotation, Annäherung usw.) interagiert werden. Dabei wird die Funktion angeboten, das Objekt zu sperren. D.h., dass das 3-D-Modell losgelöst vom Marker angezeigt wird und die

\footnotetext{
4 Weitere Informationen zur Strategie zur Digitalisierung in Studium und Lehre der UDE sind unter nachfolgendem Link zu finden: https://www.uni-due.de/e-learning/digitalisierungsstrategie.php (abgerufen am 15.07.2021).
} 

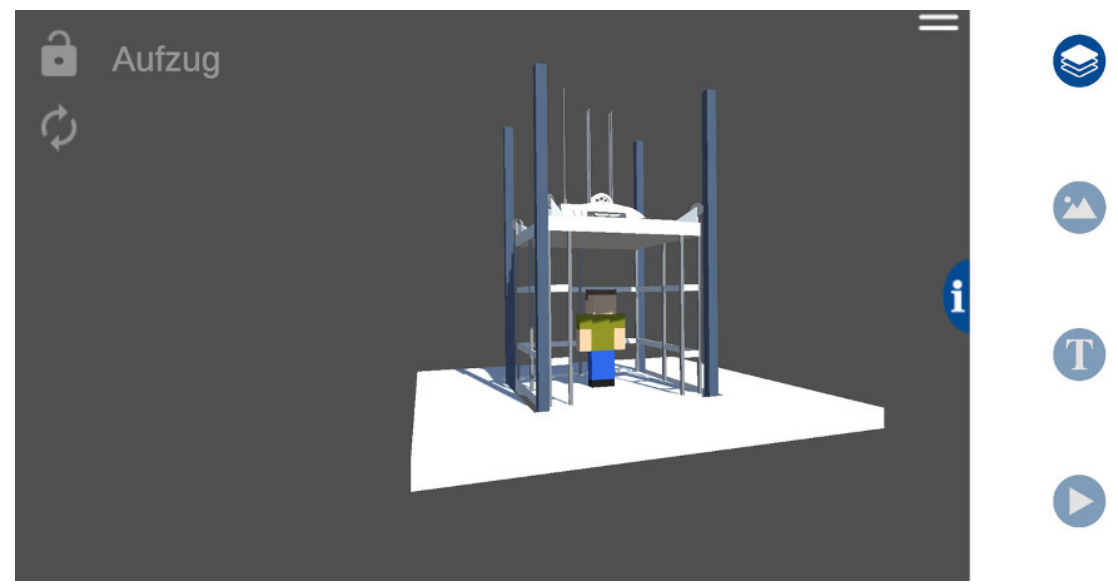

Abb. 1 Sperr-Modus und Menü für weiterführende Lerninhalte

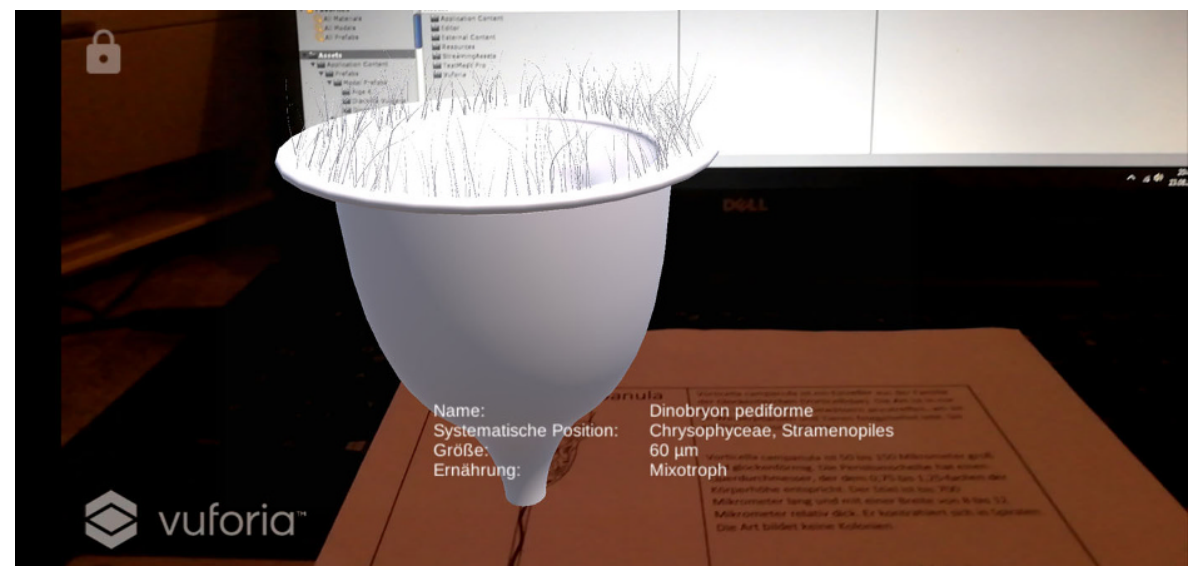

Abb. 2 Screenshot aus der App „Augmented Learning“

Interaktionsparadigmen von der Kamera auf die Gestensteuerung des Endgerätes übergehen. Dieser Sperr-Modus (s. auch Abb. 1) soll den Lernenden u. a. größtmögliche Freiheit beim Erkunden des 3-D-Modells bieten. Die Entsperrung würde zurück zur (leeren) Kameraaufnahme führen. Ist ein initiales Objekt gesperrt, wird ein Inhaltsmenü angeboten, das speziell auf weiterführendes multimediales Material verweist und direkt in der App angezeigt werden kann. Durch die interne Anzeige korrespondierender weiterer Informationen wird ein Medienbruch verhindert, wobei die Inhalte entsprechend dem mobilen Endgerät (kleiner Bildschirm usw.) anzupassen sind. Als Materialtypen können hier formatierte Texte (inkl. Hyperlinks), Abbildungen, Videos sowie weitere 3-D-Modelle referenziert werden.

Die konkrete Entwicklung der AR-Anwendung startete im Frühjahr 2019. Durch den mittlerweile vorliegenden ersten lauffähigen Prototyp ist es möglich, entsprechende Strukturen (z.B. Modelle von Algen) dreidimensional und interaktiv zu 
erkunden. Beispielhaft kann so eine vollständig modellierte Alge initial angezeigt werden (s. Abb. 2). Im Sperr-Modus wäre es dann z.B. möglich, sich verschiedene Perspektiven bzw. Schnitte anzeigen zu lassen (z.B. die Feinstruktur einer Zelle) oder aber originale Mikroskopieaufnahmen in Form verknüpfter Bilddateien aufzurufen, um z. B. den späteren Transfer für die Arbeit im Labor zu erleichtern. Die Anwendung und ihre potenziellen Inhalte können somit relativ frei verwendet bzw. ausgestaltet werden, was den fachbereichsübergreifenden Einsatz ermöglicht. Abb. 1 zeigt den Sperr-Modus an einem Beispiel aus den Bauwissenschaften. Über das seitlich aufgeklappte Menü werden die ergänzenden Inhalte angeboten.

Das Funktionskonzept zum Sperr-Modus war eine grundlegende Eigenentwicklung im Projekt, welche auf den zuvor erhobenen Lehr-Lern-Anforderungen beruhte.

Durch die gute Dokumentation der zur Entwicklung genutzten Softwarekomponenten (v.a. Unity3D und Vuforia) entstanden beim Aufbau der grundlegenden Infrastruktur kaum nennenswerte Herausforderungen. Bei der Pilotierung der ARAnwendung in der Biologie sowie seit Ende 2020 in den Bauwissenschaften zeigt sich jedoch, dass gerade das Feld der 3-D-Visualisierung in der Umsetzung eine große Hürde darstellt. Die UDE verfügt hier aktuell über keine zentral verstetigte Expertise. Da die intendierten 3-D-Objekte erst für die Fachbereiche entwickelt werden mussten, war der Aufbau entsprechender Fähigkeiten nötig. Dieser Aspekt nahm daher die meiste Zeit in Anspruch. Möchte man ein Angebot wie AR also verstetigen, wären hier entsprechende Servicestrukturen aufzubauen. Auch muss der didaktische Einsatz einer AR-Anwendung in jedem Einzelfall ausgearbeitet werden, um ein nahtloses Zusammenspiel zwischen Lehrveranstaltungen und parallel angebotenen AR-(Selbst-)Lerneinheiten zu ermöglichen.

\section{Ausblick}

Die Entscheidungsaspekte und Hürden, welche beim Einsatz von AR-Technologien im Kontext von Studium und Lehre auftreten können, sind vielfältig. Mit dem Projekt „Augmented Learning“ ist an der UDE ein niedrigschwellig nutzbares Tool erarbeitet worden, um AR in der Lehre einsetzen zu können. Durch die Nutzung des Tools in weiteren Fächern kann fachspezifisches Feedback der (potenziellen) Anwender:innen eingeholt und weitere Modelle können gemeinsam entwickelt werden. Es bietet die Möglichkeit, interessierte Akteur:innen miteinander zu vernetzen, Synergien herzustellen und möglicherweise sogar interdisziplinäre Angebote zu schaffen. Die Einführung der AR-Anwendung soll wissenschaftlich begleitet und die Erkenntnisse sollen für die Verbesserung der Anwendung genutzt werden. Auch die Untersuchung konkreter Mehrwerte für unterschiedliche AR-Lernszenarien in der Hochschule sind vorgesehen. Forschungsfragen könnten z. B. den Mehrwert von AR-Anwendungen im Rahmen der Studieneingangsphase behandeln oder die Untersuchung lernpsychologischer Ansätze, beispielsweise im Zusammengang mit dem Vorwissen der Studierenden oder der Integration von AR in spezifische Lernprozesse. Aufgrund des noch recht neuen Forschungsfeldes kann für Lehrende sowohl die Nutzung der AR-Anwendung als auch die Begleitforschung einen Reputationsge- 
winn darstellen. Ein entsprechendes Netzwerk wurde mit der Ende 2018 universitätsintern gegründeten Arbeitsgruppe „Extended Reality Learning“‘5 initiiert.

Neben der perspektivischen inhaltlichen Auseinandersetzung soll auch die technologische Weiterentwicklung der AR-App vorangetrieben werden. So ist neben der Optimierung der grafischen Benutzerschnittstelle die Umsetzung eines Autorenwerkzeugs geplant. Dies soll die Autonomie der Lehrenden stärken, die Anwendung in der eigenen Lehrveranstaltung einzusetzen. Ferner soll die App eine Art Kursverwaltung erhalten, die es ermöglicht, über einen zentralen, universitätsinternen Server Kurse bzw. „Inhaltspakete“ verwalten und bei Abruf durch die Nutzer:innen bereitstellen zu können. So wäre es beispielsweise möglich, eine Kurs-ID in der App mit optionalem Kurspasswort einzugeben und darüber die entsprechenden Inhalte zugänglich zu machen. So ließen sich veranstaltungsbezogene Distributionen der App grundsätzlich umgehen bzw. wäre eine verbesserte Differenzierung zwischen Veranstaltungen und korrespondierenden Inhalten innerhalb der App möglich. Auf diese Weise wäre die gewünschte Breitenwirkung der bislang entwickelten AR-App „Augmented Learning“ mit deutlich geringerem Personaleinsatz in der Inhaltspflege möglich.

Funding Open Access funding enabled and organized by Projekt DEAL.

Open Access Dieser Artikel wird unter der Creative Commons Namensnennung 4.0 International Lizenz veröffentlicht, welche die Nutzung, Vervielfältigung, Bearbeitung, Verbreitung und Wiedergabe in jeglichem Medium und Format erlaubt, sofern Sie den/die ursprünglichen Autor(en) und die Quelle ordnungsgemäß nennen, einen Link zur Creative Commons Lizenz beifügen und angeben, ob Änderungen vorgenommen wurden.

Die in diesem Artikel enthaltenen Bilder und sonstiges Drittmaterial unterliegen ebenfalls der genannten Creative Commons Lizenz, sofern sich aus der Abbildungslegende nichts anderes ergibt. Sofern das betreffende Material nicht unter der genannten Creative Commons Lizenz steht und die betreffende Handlung nicht nach gesetzlichen Vorschriften erlaubt ist, ist für die oben aufgeführten Weiterverwendungen des Materials die Einwilligung des jeweiligen Rechteinhabers einzuholen.

Weitere Details zur Lizenz entnehmen Sie bitte der Lizenzinformation auf http://creativecommons.org/ licenses/by/4.0/deed.de.

\section{Literatur}

Bower M, Howe C, McCredie N, Robinson A, Grover D (2014) Augmented reality in education-cases, places and potentials. EMI Educ Media Int 51:1-15

EFI - Expertenkommission Forschung und Innovation (2019) Gutachten zu Forschung, Innovation und technologischer Leistungsfähigkeit Deutschlands 2019. EFI, Berlin

Hochschulforum Digitalisierung (2016) Zur nachhaltigen Implementierung von Lerninnovationen mit digitalen Medien. Hochschulforum Digitalisierung, Berlin

Joan R (2015) Enhancing education through mobile augmented reality. I-manager's J Educ Technol 11(4):8-14

Johnson L, Adams Becker S, Cummins M, Estrada V, Freeman A, Hall C (2016) NMC horizon report: 2016 higher education edition. The New Media Consortium, Austin

Kleimann B, Wannemacher K (2004) E-Learning an deutschen Hochschulen. Von der Projektentwicklung zur nachhaltigen Implementierung. Hochschulplanung, Bd. Band 165. HIS, Hannover

Lee K (2012) Augmented reality in education and training. TechTrends 56:13-21

\footnotetext{
5 Siehe: https://www.uni-due.de/e-learning/ag-xr-learning.php (abgerufen am 27.10.2021).
} 
Mayer RE (2001) Mulitmedia learning. Cambridge University Press, Cambridge

Niedermeier S, Müller-Kreiner C (2019) VR/AR in der Lehre!? Eine Übersichtsstudie zu Zukunftsvisionen des digitalen Lernens aus der Sicht von Studierenden (URN: urn:nbn:de:0111-pedocs-180489)

Paivio A (1986) Dual coding theory. In: Paivio A (Hrsg) Mental representations: a dual coding approach. Oxford University Press, Oxford, New York, Toronto, S 53-83

Radu I (2014) Augmented Reality in education: a meta-review and cross-media analysis. Pers Ubiquit Comput 18:1533-1543

Reinmann G (2005) Lernort Universität? E-Learning im Schnittfeld von Strategie und Kultur. Z Hochschuldidaktik 6:66-84

Seufert S, Euler D (2004) Nachhaltigkeit von eLearning-Innovationen. Ergebnisse einer Dephie-Studie. Swiss Competence Centre for Innovations in Learning, Universität St. Gallen, St. Gallen

Sweller J (1994) Cognitive load theory, learning difficulty, and instructional design. Learn Instr 4:295-312

Thees M, Kapp S, Strzys MP, Beil F, Lukowicz P, Kuhn J (2020) Effects of augmented reality on learning and cognitive load in university physics laboratory courses. Comput Human Behav. https://doi.org/ 10.1016/j.chb.2020.106316

Wu H-K, Wen-Yu Lee S, Hsin-Yi C, Jyh-Chong L (2013) Current status, opportunities and challenges of augmented reality in eduction. Comput Educ 62:41-49

Yuen S, Yaoyuneyong G, Johnson E (2011) Augmented Reality: An overview and five directions for AR in education. J Educ Technol Dev Exch 4:119-140

Zender R, Weise M, von der Heyde M, Söbke H (2018) Lehren und Lernen mit VR und AR - Was wird. http://ceur-ws.org/Vol-2250/WS_VRAR_paper5.pdf. Zugegriffen: 07.2021 (Daniel Schiffner (Hrsg) Proceedings of DeLFI Workshops 2018, co-located with 16th e-Learning Conference of the German Computer Society (DeLFI 2018), Frankfurt, Germany, September 10, 2018)

Zick M (o. J.) Augmented Learning (Arbeitstitel). Eine generische Augmented Reality App für die Hochschullehre. https://www.uni-due.de/e-learning/ag-xr-learning.php\#augmentedlearning. Zugegriffen: 16. Juli 2021 